\title{
The Vertical Structures of Accretion Disks in AGN
}

\author{
H. Riffert ${ }^{1}$, T. Dörrer ${ }^{2}$, R. Staubert ${ }^{2}$, H. Ruder ${ }^{1}$ \\ ${ }^{1}$ Theoretische Astrophysik, 72076 Tübingen, Germany \\ ${ }^{2}$ Astronomisches Institut, 72076 Tübingen, Germany
}

Radiation emitted from an accretion disk around a central black hole is the widely accepted model for the observed optical to UV emission from AGN. We have calculated the properties of a standard $\alpha$-accretion disk (Shakura and Sunyaev, 1973). We present a fully self-consistent model of the structure and the spectrum of such a disk, i.e. the internal vertical density and temperature profiles are calculated simultaneously with the local spectra. Constant density models have been presented by (Ross et al., 1992). The central object is assumed to be a Kerr black hole (BH); relativistic corrections are included. The local energy production is assumed to be entirely due to turbulence. The radiative transfer equation is solved using the Eddington approximation. Inelastic Compton scattering is treated approximately by the Kompaneets equation, and the absorption cross section contains free-free and bound-free processes for hydrogen. The energy transport includes radiation and convection, and the convective flux is calculated in the mixing length theory, taking into account the heating and cooling of the rising elements. Although the convective flux is energetically negligible it has a strong influence on the vertical density structure. We performed several calculations for different parameters such as $\dot{M}$ and $\alpha$. In regions, where the surface radiation flux is large, we get a strong density inversion because the radiation force per unit mass overcomes the gravitational force. Such a density profile, however, is unstable against convection. Including the convective flux then leads to a monotonic density profile. Figures 1 and 2 show the structure and integrated spectrum for two different disk models.
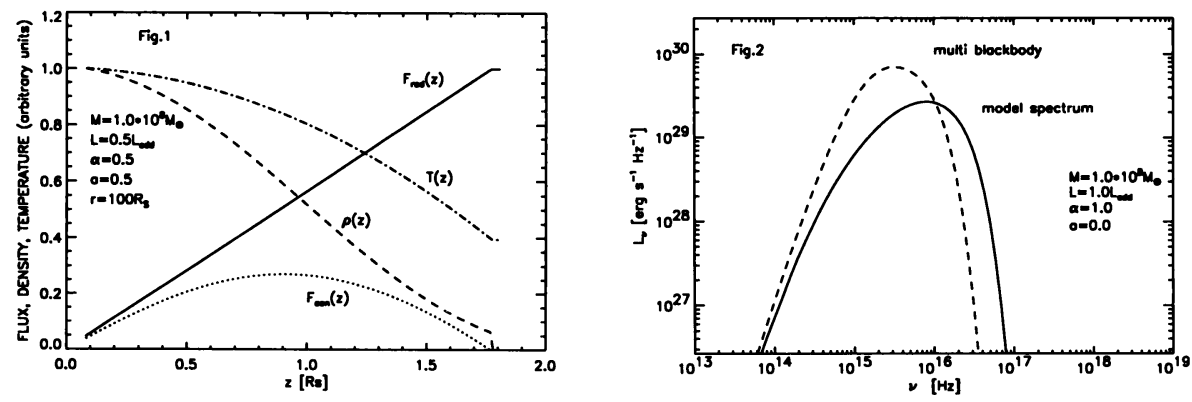

Fig. 1. Vertical disk structure for a fixed radius $r, \mathrm{BH}$ mass $M$, luminosity $L$, viscosity $\alpha$, and angular momentum $a . \rho, T$ are normalized to their central values $\rho_{0}=6.3 * 10^{-12} \mathrm{~g} / \mathrm{cm}^{3}$, $T_{0}=1.7 * 10^{5} \mathrm{~K}$; the fluxes are in units of $F_{0}=2.1 * 10^{15} \mathrm{erg} / \mathrm{cm}^{2} / \mathrm{s}$. The energy production is proportional to the total pressure.

Fig. 2. Integrated spectrum for a disk with an energy production proportional to the gas pressure only.

\section{References}

Ross, R.R., Fabian, A.C. and Mineshige, S.: 1992, Mon.Not.R.astr.Soc.258,189.

Shakura, N.I. and Sunyaev, R.A.: 1973, Astr.Ap.24, 337.

T. J.-L. Courvoisier and A. Blecha: Multi-Wavelength Continuum Emission of AGN, 478.

(C) 1994 IAU. Printed in the Netherlands. 